\title{
Approximation of a symmetric $\alpha$-stable Lévy process by a Lévy process with finite moments of all orders
}

\author{
by
}

\author{
Z. Michna (Wrocław)
}

\begin{abstract}
In this paper we consider a symmetric $\alpha$-stable Lévy process $Z$. We use a series representation of $Z$ to condition it on the largest jump. Under this condition, $Z$ can be presented as a sum of two independent processes. One of them is a Lévy process $Y_{x}$ parametrized by $x>0$ which has finite moments of all orders. We show that $Y_{x}$ converges to $Z$ uniformly on compact sets with probability one as $x \downarrow 0$. The first term in the cumulant expansion of $Y_{x}$ corresponds to a Brownian motion which implies that $Y_{x}$ can be approximated by Brownian motion when $x$ is large. We also study integrals of a nonrandom function with respect to $Y_{x}$ and derive the covariance function of those integrals. A symmetric $\alpha$-stable random vector is approximated with probability one by a random vector with components having finite second moments.
\end{abstract}

1. Introduction. Stable Lévy processes play an important role among stable processes, similar to that of Brownian motion among Gaussian processes. Thus we start with the definition of a symmetric $\alpha$-stable Lévy process (for a comprehensive treatment of stable variables and processes see e.g. Janicki and Weron [2] or Samorodnitsky and Taqqu [8]). We will consider stochastic processes on the time interval $[0,1]$.

Definition 1.1. A stochastic process $\{Z(t), 0 \leq t \leq 1\}$ is called a symmetric $\alpha$-stable Lévy process $(0<\alpha \leq 2)$ if

1. $Z(0)=0$ a.s.

2. $Z$ has independent increments.

3. $Z(t)-Z(s)$ has distribution $S_{\alpha}\left(\sigma(t-s)^{1 / \alpha}, 0,0\right)$ for any $0 \leq s<t \leq 1$, that is, $\alpha$-stable distribution with scale parameter $\sigma(t-s)^{1 / \alpha}$, and skewness and shift parameters equal to zero.

For $\alpha=2$ we get Brownian motion. If the first two assumptions are satisfied and the process has stationary increments we call it a Lévy process. In this paper we will consider $0<\alpha<2$.

2000 Mathematics Subject Classification: Primary 60G51; Secondary 60G52.

Key words and phrases: symmetric $\alpha$-stable Lévy process, integral with respect to a symmetric $\alpha$-stable Lévy process, Lévy-Itô integral representation, series representation. 
The Lévy-Itô integral representation for a symmetric Lévy process $Y$ is the following:

$$
Y(t)=\int_{\mathbb{R} \backslash 0} y N_{t}(d y)
$$

where $N$ is the point process of jumps of $Y: N=\sum_{s: \Delta Y(s) \neq 0} \delta_{(s, \Delta Y(s))}$ (see e.g. Kallenberg [3]). $N$ is a Poisson point process with the mean measure $d s \times \nu(d y)$ where $\nu(d y)$ is a Lévy measure on $\mathbb{R} \backslash 0$. Series representations for Lévy processes can be derived from the Lévy-Itô integral representation (see Rosiński [7]). The series representation for $\alpha$-stable Lévy processes is called LePage's representation (see LePage [4] or Samorodnitsky and Taqqu [8]).

2. The series representation of a symmetric $\alpha$-stable Lévy process. A symmetric $\alpha$-stable Lévy process can be represented as the following series:

$$
Z(t)=\sigma C_{\alpha}^{1 / \alpha} \sum_{k=1}^{\infty} \Gamma_{k}^{-1 / \alpha} \gamma_{k} \boldsymbol{I}\left\{U_{k} \leq t\right\}
$$

where $0 \leq t \leq 1$ and $\left\{\Gamma_{k}\right\}_{k=1}^{\infty}$ is a sequence of arrival epochs in a Poisson process with unit arrival rate, $\left\{\gamma_{k}\right\}_{k=1}^{\infty}$ is a sequence of iid random variables satisfying

$$
\mathbb{P}\left(\gamma_{k}=1\right)=\mathbb{P}\left(\gamma_{k}=-1\right)=1 / 2,
$$

and $\left\{U_{k}\right\}_{k=1}^{\infty}$ is a sequence of iid random variables uniformly distributed on $[0,1]$. These sequences are independent and

$$
C_{\alpha}=\left(\int_{0}^{\infty} s^{-\alpha} \sin s d s\right)^{-1}= \begin{cases}\frac{1-\alpha}{\Gamma(2-\alpha) \cos (\pi \alpha / 2)} & \text { if } \alpha \neq 1 \\ 2 / \pi & \text { if } \alpha=1\end{cases}
$$

A symmetric $\alpha$-stable Lévy process can be regarded as a pure jump process. The epochs of its jumps are described by $\left\{U_{k}\right\}_{k=1}^{\infty}$, the direction of a jump is governed by $\left\{\gamma_{k}\right\}_{k=1}^{\infty}$ and the heights of the jumps are presented in decreasing order by $\left\{\sigma C_{\alpha}^{1 / \alpha} \Gamma_{k}^{-1 / \alpha}\right\}_{k=1}^{\infty}$.

Let us introduce the Poisson random measure and the Lévy measure generated by the process $Z$. Define

$$
N_{t}(A)=N([0, t], A)=\sum_{0 \leq s \leq t} \boldsymbol{I}_{A}\left(\Delta Z_{s}\right)
$$

for $0 \leq t \leq 1$ and any Borel subset $A$ of $\mathbb{R}$ such that $0 \notin \bar{A}$. The process $N_{t}(A), 0 \leq t \leq 1$, is a Poisson process with arrival rate

$$
\nu(A)=\mathbb{E} N_{1}(A) .
$$

The measure $\nu$ is called the Lévy measure of the Lévy process $Z$. In fact the measure $N([0, t], A)$ can be extended to a Poisson measure on $[0,1] \times \mathbb{R} \backslash 0$ 
with the mean measure $d s \times \nu(d y)$. Thus using the series representation we obtain

$$
N_{t}(A)=\sum_{k=1}^{\infty} \boldsymbol{I}_{A}\left\{\sigma C_{\alpha}^{1 / \alpha} \Gamma_{k}^{-1 / \alpha} \gamma_{k}\right\} \boldsymbol{I}\left\{U_{k} \leq t\right\}
$$

and

$$
\nu(A)=\sum_{k=1}^{\infty} \mathbb{E} \boldsymbol{I}_{A}\left\{\sigma C_{\alpha}^{1 / \alpha} \Gamma_{k}^{-1 / \alpha} \gamma_{k}\right\} .
$$

This Lévy measure has the following form:

$$
\nu(d y)=\frac{P}{y^{1+\alpha}} \boldsymbol{I}_{(0, \infty)}(y) d y+\frac{P}{|y|^{1+\alpha}} \boldsymbol{I}_{(-\infty, 0)}(y) d y
$$

where

$$
P=\frac{1}{2} \alpha \sigma^{\alpha} C_{\alpha}
$$

3. Conditioning on the largest jump. Consider a symmetric $\alpha$ stable Lévy process under the condition $\Gamma_{1}=x$ where $x>0$ ( $\Gamma_{1}$ has exponential distribution with parameter 1). Then we get

$$
\begin{aligned}
Z_{x}(t) \stackrel{d}{=} & \sigma C_{\alpha}^{1 / \alpha} x^{-1 / \alpha} \gamma_{1} \boldsymbol{I}\left\{U_{1} \leq t\right\} \\
& +\sigma C_{\alpha}^{1 / \alpha} \sum_{k=1}^{\infty}\left(\Gamma_{k}+x\right)^{-1 / \alpha} \gamma_{k+1} \boldsymbol{I}\left\{U_{k+1} \leq t\right\} .
\end{aligned}
$$

Therefore let us investigate the following process:

$$
\begin{gathered}
Y_{x}(t)=\sigma C_{\alpha}^{1 / \alpha} \sum_{k=1}^{\infty}\left(\Gamma_{k}+x\right)^{-1 / \alpha} \gamma_{k+1} \boldsymbol{I}\left\{U_{k+1} \leq t\right\} \\
\stackrel{d}{=} \sigma C_{\alpha}^{1 / \alpha} \sum_{k=1}^{\infty}\left(\Gamma_{k}+x\right)^{-1 / \alpha} \gamma_{k} \boldsymbol{I}\left\{U_{k} \leq t\right\}
\end{gathered}
$$

and note that

$$
Z_{x}(t) \stackrel{d}{=} A_{x}(t)+Y_{x}(t)
$$

where $A_{x}(t)=\sigma C_{\alpha}^{1 / \alpha} x^{-1 / \alpha} \gamma_{1} \boldsymbol{I}\left\{U_{1} \leq t\right\}$ and the processes $A_{x}$ and $Y_{x}$ are independent.

Proposition 3.1. The process $Y_{x}$ is well-defined, that is, the sum in (9) converges almost surely.

Proof. This is a special case of Proposition 3.3 below.

Let us define the following auxiliary process:

$$
Y_{x, z}(t)=\sigma C_{\alpha}^{1 / \alpha} \sum_{k=1}^{\infty}\left(\Gamma_{k}+x\right)^{-1 / \alpha} \gamma_{k} \boldsymbol{I}\left\{U_{k} \leq t\right\} \boldsymbol{I}\left\{\Gamma_{k}<z\right\} .
$$


Notice that the series in (11) consists of finite random number of nonzero terms a.s. (by the strong law of large numbers for the sums $\Gamma_{k}$ ) and $Y_{x, z}(t) \rightarrow$ $Y_{x}(t)$ a.s. as $z \rightarrow \infty$ for all $0 \leq t \leq 1$. The process (11) can be written using the Poisson measure $N$ defined in (4) in the following way:

$$
Y_{x, z}(t)=\int_{A_{z}} f_{x}(y) N_{t}(d y)
$$

where $A_{z}=\left(-\infty,-z^{-1 / \alpha}\right) \cup\left(z^{-1 / \alpha}, \infty\right)$ and (for simplicity we assume here that $\left.\sigma C_{\alpha}^{1 / \alpha}=1\right)$

$$
f_{x}(y)=\frac{1}{\left(|y|^{-\alpha}+x\right)^{1 / \alpha}} \operatorname{sgn}(y) .
$$

It follows that $Y_{x, z}$ is a Lévy process (see e.g. Protter [6, Th. 36]).

THeOREM 3.1. The process $Y_{x}$ defined in (9) is a Lévy process with finite moments of all orders and

$$
Y_{x}(t)=\int_{\mathbb{R} \backslash 0} f_{x}(y) N_{t}(d y)
$$

in the sense of the limit of $Y_{x, z}(t)$ given in (12) as $z \rightarrow \infty$ a.s.

Proof. The stationarity of the increments follows from the fact that

$$
\boldsymbol{I}\left\{U_{k} \leq t\right\}-\boldsymbol{I}\left\{U_{k} \leq s\right\}=\boldsymbol{I}\left\{s<U_{k} \leq t\right\} \stackrel{d}{=} \boldsymbol{I}\left\{U_{k} \leq t-s\right\}
$$

for all $0 \leq s<t \leq 1$. From the convergence $Y_{x, z}(t) \rightarrow Y_{x}(t)$ a.s. as $z \rightarrow \infty$ for all $0 \leq t \leq 1$ we obtain the independence of the increments of $Y_{x}$. Since $Y_{x}$ has bounded jumps, it has finite moments of all orders (see Protter [6, Th. 34]).

We derive the exact form of the Lévy measure of the process $Y_{x}$ and the characteristic function of the random variable $Y_{x}(t)$.

TheOREM 3.2. The Lévy measure of the process $Y_{x}$ has the form

$$
\nu_{x}(d y)=\frac{P}{y^{1+\alpha}} \boldsymbol{I}_{\left(0, \sigma C_{\alpha}^{1 / \alpha} x^{-1 / \alpha}\right)}(y) d y+\frac{P}{|y|^{1+\alpha}} \boldsymbol{I}_{\left(-\sigma C_{\alpha}^{1 / \alpha} x^{-1 / \alpha}, 0\right)}(y) d y .
$$

The characteristic function of the random variable $Y_{x}(t)$ is

$$
\begin{aligned}
\mathbb{E} \exp \left(i u Y_{x}(t)\right) & =\exp \left(-t|u|^{\alpha} 2 P \int_{0}^{|u| \sigma C_{\alpha}^{1 / \alpha} x^{-1 / \alpha}} \frac{1-\cos s}{s^{1+\alpha}} d s\right) \\
& =\exp \left(-t x \alpha \sum_{k=1}^{\infty} \frac{(-1)^{k-1}}{(2 k-\alpha)(2 k) !}\left(u \sigma C_{\alpha}^{1 / \alpha} x^{-1 / \alpha}\right)^{2 k}\right)
\end{aligned}
$$


Proof. Let $\nu_{x}$ be the Lévy measure of the process $Y_{x}$. Then

$$
\nu_{x}(A)=\sum_{k=1}^{\infty} \mathbb{E} \boldsymbol{I}_{A}\left(\sigma C_{\alpha}^{1 / \alpha}\left(\Gamma_{k}+x\right)^{-1 / \alpha} \gamma_{k}\right)
$$

for any $A \subset \mathbb{R}$ with $0 \notin \bar{A}$. For simplicity we assume that $\sigma C_{\alpha}^{1 / \alpha}=1$. Take $A=(y, \infty)$ where $y>0$ and $y<x^{-1 / \alpha}$ because $\left(\Gamma_{k}+x\right)^{-1 / \alpha}<x^{-1 / \alpha}$ a.s. Since $\left(\Gamma_{k}+x\right)^{-1 / \alpha}>y$ is equivalent to $\Gamma_{k}^{-1 / \alpha}>\left(y^{-\alpha}-x\right)^{-1 / \alpha}$, using (6), (7) and (18) we get

$$
\nu_{x}(A)=\int_{\left(y^{-\alpha}-x\right)^{-1 / \alpha}}^{\infty} \frac{P}{z^{1+\alpha}} d z=\int_{y}^{x^{-1 / \alpha}} \frac{P}{w^{1+\alpha}} d w .
$$

For $y<0$ we proceed similarly. Thus we obtain

$$
\nu_{x}(d y)=\frac{P}{y^{1+\alpha}} \boldsymbol{I}_{\left(0, x^{-1 / \alpha}\right)}(y) d y+\frac{P}{|y|^{1+\alpha}} \boldsymbol{I}_{\left(-x^{-1 / \alpha}, 0\right)}(y) d y .
$$

From the Lévy-Khinchin formula we can write

$$
\mathbb{E} \exp \left(i u Y_{x}(t)\right)=\exp (-t \psi(u))
$$

where for $u \geq 0$,

$$
\begin{aligned}
\psi(u) & =\int_{\mathbb{R} \backslash 0}\left(1-e^{i u y}\right) \nu_{x}(d y)=\int_{\mathbb{R} \backslash 0}(1-\cos (u y)) \nu_{x}(d y) \\
& =\int_{0}^{x^{-1 / \alpha}}(1-\cos (u y)) \frac{2 P}{y^{1+\alpha}} d y=2 P u^{\alpha} \int_{0}^{u x^{-1 / \alpha}} \frac{1-\cos (s)}{s^{1+\alpha}} d s
\end{aligned}
$$

where in the last equality we have substituted $s=u y$. Arguing similarly for $u<0$, we arrive at (16). To obtain the second form of the characteristic function we expand $\cos s$ in Taylor series and integrate each summand.

In the next proposition we derive the covariance structure of the process $Y_{x}$.

Proposition 3.2. Let $Y_{x}$ be defined in (9). Then

and

$$
\begin{aligned}
\mathbb{E} Y_{x}(t) & =0 \\
\mathbb{E} Y_{x}^{2}(t) & =\mathbb{E}\left(\int_{\mathbb{R} \backslash 0} f_{x}(y) N_{t}(d y)\right)^{2}=t \int_{\mathbb{R} \backslash 0} f_{x}^{2}(y) \nu(d y)=t \sigma^{2}(x)
\end{aligned}
$$

where

$$
\mathbb{E} Y_{x}(s) Y_{x}(t)=\sigma^{2}(x) \min \{s, t\}
$$

$$
\sigma^{2}(x)=\frac{\alpha \sigma^{2} C_{\alpha}^{2 / \alpha}}{(2-\alpha) x^{(2-\alpha) / \alpha}} .
$$


Proof. The expectation is zero because the process $Y_{x}$ is symmetric. The form of the variance follows from the well known fact that for Lévy processes without Gaussian component, $\operatorname{Var} Y_{x}(t)=t \int y^{2} \nu_{x}(d y)$. (Twice differentiating the characteristic function in the form (17) also gives the second moment of the random variable $Y_{x}(t)$ in the form (20).) To get the desired form of covariance function we use the equality $Y(s) Y(t)=Y(s)(Y(t)-Y(s))+Y^{2}(s)$ for $s<t$.

REMARK 3.1. Taking only the first term in the series (17) we obtain the characteristic function of Brownian motion $B_{x}$ with variance $\sigma^{2}(x)=$ $\frac{\alpha \sigma^{2} C_{\alpha}^{2 / \alpha}}{(2-\alpha) x^{(2-\alpha) / \alpha}}$ the same as that of $Y_{x}$, that is,

$$
\mathbb{E} \exp \left(i u B_{x}(t)\right)=\exp \left(-\frac{t u^{2}}{2} \frac{\alpha \sigma^{2} C_{\alpha}^{2 / \alpha}}{(2-\alpha) x^{(2-\alpha) / \alpha}}\right) .
$$

Now we investigate the integral of a non-random measurable function $g$ with respect to the process $Y_{x}$, that is,

$$
\int_{0}^{1} g(s) d Y_{x}(s)
$$

We impose assumptions on $g$ to ensure that the second moment of the integral is finite. The integral of a non-random function $g$ with respect to a symmetric $\alpha$-stable Lévy process and the process $Y_{x}$ is well defined if $g \in L^{\alpha}([0,1], d s)$ (this follows from Urbanik and Woyczyński [9]).

THEOREM 3.3. If the functions $g^{2}$ and $h^{2}$ are integrable on $[0,1]$ then

$$
\begin{gathered}
\mathbb{E}\left(\int_{0}^{1} g(s) d Y_{x}(s)\right)=0 \\
\mathbb{E}\left(\int_{0}^{1} g(s) d Y_{x}(s)\right)^{2}=\sigma^{2}(x) \int_{0}^{1} g^{2}(s) d s,
\end{gathered}
$$

and

$$
\mathbb{E}\left(\int_{0}^{1} g(s) d Y_{x}(s)\right)\left(\int_{0}^{1} h(s) d Y_{x}(s)\right)=\sigma^{2}(x) \int_{0}^{1} g(s) h(s) d s .
$$

Proof. Since $Y_{x}$ is a square integrable martingale, by the Doob inequality we are able to define the above integrals in $L^{2}$ sense and get the desired equalities (see e.g. Kallenberg [3]).

In fact the integral $\int_{0}^{1} g(s) d Y_{x}(s)$ can be defined for $g \in L^{\alpha}([0,1], d s)$ in the following way:

$$
\int_{0}^{1} g(s) d Y_{x}(s)=\sigma C_{\alpha}^{1 / \alpha} \sum_{k=1}^{\infty}\left(\Gamma_{k}+x\right)^{-1 / \alpha} \gamma_{k} g\left(U_{k}\right) .
$$


Proposition 3.3. If $g \in L^{\alpha}([0,1], d s)$ then the series in $(24)$ is convergent a.s.

Proof. This is an easy application of [7, Th. 4.1].

REMARK 3.2. If $g \in L^{2}([0,1], d s)$ then the integral $\int_{0}^{1} g(s) d Y_{x}(s)$ defined in (24) coincides (in the finite-dimensional distribution sense) with the $L^{2}$ integral appearing in Theorem 3.3.

4. Approximation of a symmetric $\alpha$-stable Lévy process and Brownian motion. The process $Y_{x}$ approximates the symmetric $\alpha$-stable Lévy process $Z$.

THEOREM 4.1. The process $Y_{x}$ converges uniformly on compact sets to the process $Z$ with probability one as $x \downarrow 0$; more precisely,

$$
\sup _{0 \leq t \leq 1}\left|Y_{x}(t)-Z(t)\right| \leq x \alpha^{-1} \sigma C_{\alpha}^{1 / \alpha} Z_{\alpha /(\alpha+1)}
$$

a.s. for $x \geq 0$, where

$$
Z_{\alpha /(\alpha+1)}=\sum_{k=1}^{\infty} \frac{1}{\Gamma_{k}^{(\alpha+1) / \alpha}}
$$

is an $\alpha /(\alpha+1)$-stable random variable with skewness parameter one and shift parameter zero.

Proof. Consider the function $g(x)=a^{-1 / \alpha}-(a+x)^{-1 / \alpha}$ for fixed $a>0$ and $x \geq 0$. Since

$$
\frac{d}{d x}\left[a^{-1 / \alpha}-(a+x)^{-1 / \alpha}\right]=\frac{1}{\alpha(a+x)^{1+1 / \alpha}} \leq \frac{1}{\alpha a^{1+1 / \alpha}}
$$

it is easy to notice that

$$
\left|a^{-1 / \alpha}-(a+x)^{-1 / \alpha}\right| \leq \frac{1}{\alpha a^{1+1 / \alpha}} x
$$

Thus we obtain

$$
\begin{aligned}
\sup _{0 \leq t \leq 1} \sigma C_{\alpha}^{1 / \alpha}\left|\sum_{k=1}^{\infty}\left[\Gamma_{k}^{-1 / \alpha}-\left(\Gamma_{k}+x\right)^{-1 / \alpha}\right] \gamma_{k} \boldsymbol{I}\left\{U_{k} \leq t\right\}\right| \\
\leq \sigma C_{\alpha}^{1 / \alpha} \sum_{k=1}^{\infty}\left|\Gamma_{k}^{-1 / \alpha}-\left(\Gamma_{k}+x\right)^{-1 / \alpha}\right| \leq \frac{x}{\alpha} \sigma C_{\alpha}^{1 / \alpha} \sum_{k=1}^{\infty} \frac{1}{\Gamma_{k}^{1+1 / \alpha}} \\
\quad=\frac{x}{\alpha} \sigma C_{\alpha}^{1 / \alpha} \sum_{k=1}^{\infty} \frac{1}{\Gamma_{k}^{(\alpha+1) / \alpha}}=\frac{x}{\alpha} \sigma C_{\alpha}^{1 / \alpha} Z_{\alpha /(\alpha+1)}
\end{aligned}
$$

where $Z_{\alpha /(\alpha+1)}$ is an $\alpha /(\alpha+1)$-stable random variable with skewness parameter one and shift parameter zero (the series is a.s. convergent, see Samorodnitsky and Taqqu [8]). 
Similarly we can prove the following proposition.

Proposition 4.1. Let

$$
X(t)=\int_{0}^{1} g_{t}(s) d Y_{x}(s)=\sigma C_{\alpha}^{1 / \alpha} \sum_{k=1}^{\infty}\left(\Gamma_{k}+x\right)^{-1 / \alpha} \gamma_{k} g_{t}\left(U_{k}\right)
$$

where $g_{t}(s)$ is uniformly bounded. Then

$$
\int_{0}^{1} g_{t}(s) d Y_{x}(s) \rightarrow \int_{0}^{1} g_{t}(s) d Z(s)
$$

uniformly on compact sets a.s. as $x \downarrow 0$.

Let us consider a symmetric $\alpha$-stable random vector and recall the following representation theorem (see Samorodnitsky and Taqqu [8]).

THEOREM 4.2. If $X$ is a symmetric $\alpha$-stable random vector then there are bounded measurable functions $g_{1}, \ldots, g_{d}$ such that

$$
X \stackrel{d}{=}\left(\int_{0}^{1} g_{1}(s) d Z(s), \ldots, \int_{0}^{1} g_{d}(s) d Z(s)\right) .
$$

Thus we are able to approximate symmetric $\alpha$-stable random vectors by vectors with finite second moments.

TheOREM 4.3. Let $g_{1}, \ldots, g_{d}$ be bounded measurable functions. Then

$$
\begin{aligned}
X_{x} & =\left(\int_{0}^{1} g_{1}(s) d Y_{x}(s), \ldots, \int_{0}^{1} g_{d}(s) d Y_{x}(s)\right) \\
& \rightarrow\left(\int_{0}^{1} g_{1}(s) d Z(s), \ldots, \int_{0}^{1} g_{d}(s) d Z(s)\right)=X
\end{aligned}
$$

a.s. as $x \downarrow 0$ and

$$
\left\|X_{x}-X\right\|_{d} \leq \frac{x}{\alpha} M \sigma C_{\alpha}^{1 / \alpha} Z_{\alpha /(\alpha+1)}
$$

for $x \geq 0$, where $\|\cdot\|_{d}$ is Euclidean norm, $Z_{\alpha /(\alpha+1)}$ is the random variable defined in (26) and $M=\sqrt{\sum_{k=1}^{d} \sup _{0 \leq s \leq 1}\left|g_{k}(s)\right|^{2}}$.

Proof. Since the functions $g_{l}$ are bounded they belong to $L^{\alpha}([0,1], d s)$ and the integral can be defined by (24). Similarly to the proof of Theorem 4.1 we can write

$$
\begin{aligned}
& \left|\sum_{k=1}^{\infty}\left[\Gamma_{k}^{-1 / \alpha}-\left(\Gamma_{k}+x\right)^{-1 / \alpha}\right] \gamma_{k} g_{l}\left(U_{k}\right)\right| \\
\leq & M_{l} \sum_{k=1}^{\infty}\left|\Gamma_{k}^{-1 / \alpha}-\left(\Gamma_{k}+x\right)^{-1 / \alpha}\right| \leq M_{l} \frac{x}{\alpha} \sum_{k=1}^{\infty} \frac{1}{\Gamma_{k}^{(\alpha+1) / \alpha}}=M_{l} \frac{x}{\alpha} Z_{\alpha /(\alpha+1)}
\end{aligned}
$$

for $x \geq 0$, where $\sup _{0 \leq s \leq 1}\left|g_{l}(s)\right|=M_{l}$ and $Z_{\alpha /(\alpha+1)}$ is defined in (26). 
REMARK 4.1. Since $g_{1}, \ldots, g_{d}$ are bounded, they belong to $L^{2}$ and by Theorem 3.3 the components of $X_{x}$ have finite second moments.

The next question is what happens with the processes $Y_{x}$ when $x \rightarrow \infty$. The answer is the following.

THEOREM 4.4.

$$
\sqrt{\frac{2-\alpha}{\alpha \sigma^{2} C_{\alpha}^{2 / \alpha}}} x^{1 / \alpha-1 / 2} Y_{x} \Rightarrow B
$$

as $x \rightarrow \infty$ weakly in the Skorokhod space $D$ equipped with the uniform metric where $B$ is a standard Brownian motion.

Proof. Since $Y_{x}$ is a Lévy process, it is enough to show that $Y_{x}(1) \Rightarrow B(1)$ in distribution as $x \rightarrow \infty$ (this follows easily from [5, Th. V.19]). Taking the representation (17) of the characteristic function of $Y_{x}(t)$ we find that for

$$
u=u^{\prime} \sqrt{\frac{2-\alpha}{\alpha \sigma^{2} C_{\alpha}^{2 / \alpha}}} x^{1 / \alpha-1 / 2}
$$

the first term in the series converges to $t u^{\prime 2} / 2$ and the other terms tend to zero, which yields

$$
\mathbb{E} \exp \left(i u \sqrt{\frac{2-\alpha}{\alpha \sigma^{2} C_{\alpha}^{2 / \alpha}}} x^{1 / \alpha-1 / 2} Y_{x}(t)\right) \rightarrow \exp \left(-\frac{t u^{2}}{2}\right)
$$

as $x \rightarrow \infty$.

REMARK 4.2. The assertion of the above theorem can be obtained from Theorem 2.1 of [1] by substituting $\varepsilon=\sigma C_{\alpha}^{1 / \alpha} x^{-1 / \alpha}$.

Acknowledgements. The author would like to thank an anonymous referee who pointed out the inequality (27), which improved the inequalities (25) and (30).

\section{References}

[1] S. Asmussen and J. Rosiński, Approximation of small jumps of Lévy processes with a view towards simulation, J. Appl. Probab. 38 (2001), 482-493.

[2] A. Janicki and A. Weron, Simulation and Chaotic Behavior of $\alpha$-Stable Stochastic Processes, Dekker, New York, 1994.

[3] O. Kallenberg, Foundations of Modern Probability, Springer, New York, 1997.

[4] R. LePage, Multidimensional infinitely divisible variables and processes. II, in: Probability in Banach Spaces III, Lecture Notes in Math. 860, Springer, Berlin, 1981, 279-284.

[5] D. Pollard, Convergence of Stochastic Processes, Springer, New York, 1984.

[6] Ph. Protter, Stochastic Integration and Differential Equations, Springer, New York, 1990. 
[7] J. Rosiński, Series representations of Lévy processes from the perspective of point processes, in: Lévy Processes-Theory and Applications, O. E. Barndorff-Nielsen et al. (eds.), Birkhäuser, Boston, 2001, 401-415.

[8] G. Samorodnitsky and M. Taqqu, Non-Gaussian Stable Processes: Stochastic Models with Infinite Variance, Chapman and Hall, London, 1994.

[9] K. Urbanik and W. A. Woyczyński, A random integral and Orlicz spaces, Bull. Acad. Polon. Sci. Sér. Sci. Math. Astronom. Phys. 15 (1967), 161-169.

Department of Mathematics

Wrocław University of Economics

53-345 Wrocław, Poland

E-mail: michna@ds25.ae.wroc.pl

Received June 27, 2005

Revised version March 21, 2007 